\title{
Efecto d Baño Químico Con Ácido Cítrico y Ascórbico, Temperatura y Tiempo de Almacenamiento Sobre el Pardeamiento Enzimático en Persea Americana Mínimamente Procesada
}

\author{
Lurys Ivette Martínez Marín, (MEd) \\ Departamento de Salud Pública, Universidad Especializada de las Américas, \\ Panamá \\ Angie Macías, (Licda) \\ Departamento de Salud Pública, Universidad Especializada de las Américas, \\ Panamá
}

\section{Doi:10.19044/esj.2021.v17n37p247}

Submitted: 14 September 2021

Accepted: 30 September 2021

Published: 31 October 2021
Copyright 2021 Author(s)

Under Creative Commons BY-NC-ND 4.0 OPEN ACCESS

Cite As:

N’Guessan A.H., Gogoue D.O., Anougba B.D., Dembélé I. \& Allou K. (2021). Evaluation de Différents Types de Substrats sur Le Développement des Plantules de Palmiers À Huile (Elaeis Guineensis Jacq.) en Côte d'Ivoire. European Scientific Journal, ESJ, 17(37), 247. https://doi.org/10.19044/esj.2021.v17n37p247

\section{Résumé}

Se evaluó el efecto de los ácidos orgánicos sobre el pardeamiento enzimático de la Persea americana variedad Hass, mínimamente procesada, empacada al vacío y almacenada a distintas temperaturas. Las muestras de aguacate se trataron con un baño químico de ácidos orgánicos (ácido cítrico y ácido ascórbico) con tres concentraciones diferentes, luego se empacaron al vacío y fueron almacenadas a $4^{\circ}$ y $8^{\circ} \mathrm{C}$ durante 12 días. Muestras sin tratamiento se emplearon como control y todas las muestras se evaluaron cada $0,4,8$ y 12 días. Se concluye que a $8^{\circ} \mathrm{C}$ el color y el sabor del aguacate mejora. Durante cada observación no hubo pérdida significativa de masa. Estos resultados, aunque incipientes, son útiles porque pueden contribuir con la seguridad alimentaria, además de determinar nuevas formas de preservar Persea americana y poder comercializarla como un producto de cuarta gama.

Palabras clave: Ácidos Orgánicos, Alimentos Mínimamente Procesados, Baño Químico, Pardeamiento Enzimático 


\title{
Effects of Citric Acid and Ascorbic Acid, Temperature and Storage Time as Inhibitors of Browning in Minimally Processed Persea Americana
}

\section{Lurys Ivette Martínez Marín, (MEd)}

Departamento de Salud Pública, Universidad Especializada de las Américas, Panamá

Angie Macías, (Licda)

Departamento de Salud Pública, Universidad Especializada de las Américas, Panamá

\begin{abstract}
The effect of organic acids on the enzymatic browning of Persea americana variety Hass, minimally processed, vacuum packed and stored at different temperatures, was evaluated. The avocado samples were treated with a chemical bath of organic acids (citric acid and ascorbic acid) with three different concentrations, then they were vacuum packed and stored at $4^{\circ}$ and $8^{\circ} \mathrm{C}$ for 12 days. Untreated samples were used as control and all samples were evaluated at $0,4,8$ and 12 days. It is concluded that at $8^{\circ} \mathrm{C}$ the color and flavor of the avocado improves; the chemical bath that obtained the best results was chemical bath 3 with respect to color and the samples are best preserved on day 8. During each observation there was no significant loss of mass. These results, although incipient, are useful because they can contribute to food safety, in addition to determining new ways of preserving Persea americana and being able to market it as a fourth-range product.
\end{abstract}

Keywords: Organic Acids, Minimally Processed Foods, Chemical Bath, Browning

\section{Introducción}

El aguacate es un árbol de hoja perenne subtropical nativo de Mesoamérica que pertenece a la Lauraceae, una familia del orden Laurales que, junto con las órdenes Canellales, Piperales y Magnoliales, es incluido en el clado Magnoliid de angiospermas tempranas divergentes. Esta familia pantropical tiene alrededor de 50 géneros y de 2500 a 3000 especies (Talavera, et al., 2019).

Esta especie tiene un genoma diploide con un tamaño aproximado de $\sim 920$ Mbp y produce un fruto climatérico, carnoso y aceitoso. La floración y el cuajado son procesos que duran entre uno y tres meses, generando diferencias en las edades fisiológicas de la fruta dentro de un mismo árbol (Vergara-Pulgar et al., 2019). De acuerdo con Cortés-Herrera et al., (2019), el 
aguacate representa una industria de mil millones de dólares y sigue aumentando con el paso del tiempo, de ahí que tanto los países exportadores como los importadores se encuentren especialmente interesados en su producción y consumo debido a la alta calidad de sus nutrientes. Además de la fruta, las hojas del aguacate tienen una amplia gama de beneficios farmacológicos, tal como lo han confirmado numerosas investigaciones. Por ejemplo, se ha demostrado que el extracto acuoso de hojas de aguacate tiene un efecto analgésico y antiinflamatorio en la reducción del dolor en ratones (Loh \& Lim, 2018).

Son cinco los países que producen el 80 \% de los aguacates y México es el principal productor (Díaz, Ardila \& Guerra 2019). El consumo de aguacate a nivel mundial va en crecimiento monotónico como se puede apreciar en la tabla 1 (Arias, Montoya, \& Velásquez, 2018). De acuerdo con la FAO, la producción de aguacate en Panamá no ha cambiado, a pesar de que en el mundo la demanda del mercado ha aumentado exponencialmente (FAO, 2021).

Los productos de aguacate son inestables debido a la presencia de enzimas oxidantes que degradan su apariencia visual y sus propiedades sensoriales de color y textura. Por tanto, es necesario evaluar los procesos para obtener productos de aguacate con mínimo procesamiento (Salgado-Cervantes et al, 2019). La oxidación es provocada por la polifenol oxidasa (PPO, EC 1.14.18.1), una enzima que cataliza la hidroxilación de monofenoles a odifenoles (actividad cresolasa) y la oxidación de o-difenoles a o-quinonas (actividad catecolasa), que representan el principal producto de reacción. Las reacciones posteriores de las quinonas conducen a la acumulación de melanina, que es el pigmento marrón o negro asociado con el "pardeamiento" en las reacciones de los tejidos vegetales. La PPO se encuentra en orgánulos celulares como los cloroplastos, las mitocondrias y los peroxisomas, donde está firmemente unida a la membrana o es soluble. El ablandamiento es promovido por la pectina metilesterasa (PME, EC 3.1.1.11) y poligalacturonasa (PG, EC 3.2.1.15) (Cox et al., 2004), (Benen \& Visser, 2003).

Comprender los cambios de pigmento durante la maduración de "Hass" es fundamental ya que se podría controlar la coloración de alguna manera para coordinar mejor el suavizado y el cambio de color. Según Hernández \& Briceño (2009), la actividad de la polifenoloxidasa (PFO) se reduce de forma significativa al aplicarle un baño de ácido cítrico y ácido ascórbico a frutos y vegetales, lo que evita el proceso de pardeamiento enzimático. La aspersión con un baño químico es una técnica considerada de cuarta gama debido a la poca manipulación que se le realiza a la fruta. Se basa principalmente en la aspersión de la fruta con una solución acuosa que contiene algún compuesto químico antimicrobiano, antioxidante, 
antipardeante, entre otros (Gil-Giraldo et al., 2019). Los ácidos orgánicos poseen características antioxidantes.

Tabla No.1. Proyección del consumo per cápita aparente de aguacate en (kg-persona/año)

\begin{tabular}{|c|c|c|c|c|c|c|c|c|}
\hline Consumo per cápita & 2013 & 2014 & 2015 & 2016 & 2017 & 2018 & 2019 & 2020 \\
\hline Mundo & 0,66 & 0,71 & 0,75 & 0,74 & 0,77 & 0,80 & 0,83 & 0,85 \\
\hline Estados Unidos de América & 2,20 & 2,73 & 3,22 & 2,70 & 3,25 & 3,44 & 3,64 & 3,85 \\
\hline Países Bajos & 1,40 & 2,40 & 2,11 & 2,52 & 1,01 & 1,69 & 1,62 & 1,56 \\
\hline Francia & 1,31 & 1,54 & 1,47 & 1,73 & 1,86 & 1,97 & 2,10 & 2,22 \\
\hline Reino Unido & 0,62 & 0,81 & 1,16 & 1,49 & 1,62 & 1,94 & 2,21 & 2,48 \\
\hline España & 1,01 & 1,20 & 1,42 & 1,80 & 1,78 & 2,09 & 2,30 & 2,52 \\
\hline Canadá & 1,64 & 1,72 & 1,95 & 2,15 & 2,20 & 2,40 & 2,55 & 2,71 \\
\hline Alemania & 0,32 & 0,39 & 0,46 & 0,56 & 0,69 & 0,75 & 0,84 & 0,93 \\
\hline Japón & 0,47 & 0,45 & 0,45 & 0,58 & 0,45 & 0,50 & 0,51 & 0,52 \\
\hline China & 0,08 & 0,09 & 0,10 & 0,11 & 0,11 & 0,12 & 0,12 & 0,13 \\
\hline Bélgica & 0,55 & 0,47 & 0,51 & 0,94 & 1,22 & 1,28 & 1,46 & 1,64 \\
\hline Hong Kong, China & 0,28 & 0,48 & 0,45 & 0,70 & 0,79 & 0,91 & 1,04 & 1,16 \\
\hline Suecia & 2,04 & 1,99 & 1,82 & 1,83 & 2,09 & 1,94 & 1,93 & 1,92 \\
\hline Arabia Saudita & 0,32 & 3,34 & 0,53 & 0,54 & 0,67 & 0,75 & 0,84 & 0,93 \\
\hline Rusia, Federación de & 0,10 & 0,10 & 0,08 & 0,08 & 0,13 & 0.11 & 0.12 & 0,12 \\
\hline Argentina & 0,32 & 0,40 & 0,34 & 0,38 & 0,52 & 0,51 & 0,54 & 0,58 \\
\hline Italia & 0,09 & 0,14 & 0,17 & 0,21 & 0,26 & 0,30 & 0,34 & 0,38 \\
\hline Australia & 2,68 & 2,66 & 2,65 & 3,51 & 3,33 & 3,62 & 3,83 & 4,05 \\
\hline Emiratos Árabes Unidos & 0,79 & 1,39 & 1,54 & 1,60 & 2,04 & 2,29 & 2,56 & 2,83 \\
\hline Suiza & 0,97 & 1,16 & 1,37 & 1,65 & 1,82 & 2,05 & 2,27 & 2,48 \\
\hline Dinamarca & 1,74 & 1,91 & 2,43 & 2,31 & 2,29 & 2,59 & 2,74 & 2,89 \\
\hline Polonia & 0,10 & 0,16 & 0,21 & 0,22 & 0,30 & 0,34 & 0,39 & 0,43 \\
\hline Noruega & 1,73 & 2,04 & 2,25 & 2,36 & 2,48 & 2,72 & 2,90 & 3,08 \\
\hline México & 7,40 & 7,02 & 6,20 & 7,55 & 8,54 & 8,19 & 8,47 & 8,75 \\
\hline Dominicana & 35,69 & 39,31 & 48,55 & 54,00 & 54,50 & 62,10 & 67,33 & 72,56 \\
\hline Colombia & 6,30 & 6,20 & 6,33 & 5,99 & 6,31 & 6,17 & 6,15 & 6,13 \\
\hline Perú & 5,69 & 5,50 & 6,10 & 8,22 & 7,19 & 8,26 & 8,83 & 9,41 \\
\hline Brasil & 0,76 & 0,74 & 0,86 & 0,92 & 0,85 & 0,93 & 0,97 & 1,01 \\
\hline Kenya & 3,41 & 4,12 & 2,07 & 2,67 & 3,42 & 2,71 & 5,56 & 2,42 \\
\hline Indonesia & 1,15 & 1,20 & 1,48 & 1,17 & 1,37 & 1,40 & 1,44 & 1,48 \\
\hline
\end{tabular}

Nota: cálculos propios con datos de FAO (2021), ITC (2018)

La industria de alimentos utiliza los ácidos principalmente por su función de acidificación, toda vez que medir cuantitativamente su nivel de acidez $(\mathrm{pH})$; se observa que un bajo nivel de $\mathrm{pH}$ retardará el crecimiento de microorganismos indeseables y bacterias, de igual manera aumentará la efectividad de sus conservadores y se aminora el requerimiento de tratamientos térmicos drásticos en la esterilización de frutas y verduras 
enlatadas como que también promueve la inactivación de enzimas indeseables como polifenoloxidasas (Papagianni, 2001).

Si bien, el objetivo del presente estudio es evaluar el efecto del baño químico con ácido cítrico y ácido ascórbico, concentración, refrigeración, temperatura y pardeamiento enzimático en Persea americana mínimamente procesada, este experimento es el precursor de este tipo que se realiza en Panamá, con la expectativa de que sea la simiente para otras investigaciones donde se observen nuevas variables, con la confianza de que se pueda contribuir en el futuro próximo con la seguridad alimentaria.

\section{Materiales y método \\ Materia prima}

Para este estudio se emplearon 40 aguacates variedad Hass obtenidos de un proveedor particular, que garantizó la colecta de los mismos árboles de su huerto, con el fin de certificar el manejo del monocultivo de aguacate, la distancia de siembra, la calidad química del suelo, la topografía, la capacidad de cobertura, la cantidad de luz, recurso hídrico y nutrientes.

La entrega del material se realizó bajo las más estrictas normas al laboratorio de la universidad en el que se llevó a cabo el experimento. La madurez de consumo se registró a través de la medición de la longitud y diámetro de cada uno de ellos, además, se utilizó un colorímetro de reflectancia marca Konica Minolta, asimismo se obtuvieron los valores de luminosidad, cromaticidad y matiz.

Posteriormente a la sanitización, se procedió a trocear con un cuchillo de corte y fueron colocados en una bandeja de plástico, donde se le aplicó por aspersión el baño químico de ácidos orgánicos (Gil-Giraldo et al., 2019).

\section{Agente inhibidor}

El baño químico se preparó con agua hervida cuyo pH fue de 7.04. Para el oscurecimiento enzimático se utilizó el ácido cítrico no solamente por su comprobado funcionamiento en la industria alimentaria, sino además por el bajo costo que implica su adquisición. Asimismo, se empleó ácido ascórbico, $\left(\mathrm{C}_{6} \mathrm{H}_{8} \mathrm{O}_{6}\right)$ como agente antioxidante. La pureza de ambos ácidos es mayor al $99.7 \%$.

De acuerdo con Campos (2014), los antioxidantes ácido ascórbico y ácido cítrico suministran un control adecuado en la postcosecha, con una concentración de 3 \% y, la combinación de ambos en la proporción de 2:1.

En el experimento, las mezclas se prepararon con la unidad de concentración denominada porcentaje de concentración masa/volumen. Cada solución contenía dos ácidos: ácido ascórbico $\left(0.5 \mathrm{~g} \mathrm{~L}^{-1}, 1.0 \mathrm{~g} \mathrm{~L}^{-1}\right.$ y $\left.15 \mathrm{gL}^{-1}\right)$ y ácido cítrico (0.5g L $\mathrm{L}^{-1}$, 1.0g L $\mathrm{L}^{-1}$ y $\left.15 \mathrm{gL}-1\right)$. Es decir, el baño químico 1 (BQ 1 ) tenía $0.5 \mathrm{~g} \mathrm{~L}^{-1}$ de ácido ascórbico y $0.5 \mathrm{~g} \mathrm{~L}^{-1}$ de ácido cítrico. 


\section{Equipos}

Se lavaron y desinfectaron los instrumentos y la cristalería, con una solución de 500 ppm de hipoclorito de sodio. Se utilizaron vasos químicos de $50 \mathrm{ml}$, y matraces de preparación de $100 \mathrm{ml}$, para elaborar las soluciones acuosas de ácidos cítrico y ácido ascórbico. Las muestras fueron almacenadas en una refrigeradora marca FRIGERADE Modelo\# FFTR1817LW8 SERIE BA22121891 cuya temperatura se graduó a $4^{\circ} \mathrm{C}$ y en un enfriador Thermo Fisher Scientific High Performance Refrigerators and Freezers Model \#TSX1205SA17 SERIAL \# 1123767301191121, que fue graduado a $8^{\circ} \mathrm{C}$. Las mediciones se realizaron los días $0,4,8,12$.

\section{Método}

Se prepararon ciento ocho (108) muestras para analizar la efectividad del baño químico. Se emplearon 3 muestras por tipo de baño, para cada día observado, que hacen un total de 18 muestras (incluyendo un triplicado de grupo control para cada día observado), y a su vez, luego de ser tratadas se abrieron al cuarto, octavo y doceavo día haciendo un total de 54 muestras. Como se evaluó el efecto de la temperatura de refrigeración $\left(4^{\circ} \mathrm{C}\right.$ y $\left.8^{\circ} \mathrm{C}\right)$, en las cualidades subjetivas de las porciones luego de ser empacadas al vacío, en total se evaluaron 108 muestras. Cada fruto fue cortado en cuatro partes y fueron colocados un cuarto por muestra. Como control se utilizó la fracción sin la aplicación del baño químico. Las muestras experimentales y controles se sellaron y empacaron al vacío con una máquina modelo LP-11S con un poder de 90W, que tiene una presión de vaciado de unos -60kPpa.

\section{Empacado}

Se utilizó un envasado al vació con la intención de inhibir bacterias y otros organismos que pudieran perjudicar las muestras, es decir, a través de calidad e inocuidad de alimentos. El empaque se realizó con bolsas especializadas para productos alimenticios, elaboradas con un $95 \%$ de polietileno, y un 0,5 \% de poliamida. La dimensión de las bolsas fue de $25 \mathrm{X}$ $30 \mathrm{~cm}$. La selección del material de empacado, LDPE, y el equipo de envasado al vacío, se usaron en función de resultados previos por demostrar su efectividad en el empacado (Xiao \& Kiyota, 2000).

\section{Cualidades subjetivas}

Luego de retirados del almacenamiento, los aguacates fueron inspeccionados y examinados visualmente por un panel de 9 participantes seleccionados de manera probabilística aleatorio simple y con doble ciego, (evitando el contacto o comunicación entre sí, antes y durante el experimento) para detectar el desarrollo de moho o descomposición. Se determinó el color de la piel en términos de textura, olor, sabor y color. Previo a ser cortados y luego de ser 
tratados y almacenados, se podía percibir el color de la piel como verde, o negro, sinónimo de grado de maduración (Hassan \& Dann, 2019). Los cambios en la firmeza se inspeccionaron al tacto en busca de puntos blandos (Hassan \& Dann, 2019). También se observaron y registraron otras variaciones con respecto a la apariencia física, como manchas oscuras o hematomas. Con base en las observaciones, a cada fruta se le asignó una valoración general de "buena", "regular" o "mala".

\section{Resultados}

El registro de los datos se realizó a través de la elaboración de una tabla en Excel en la que se integraron los resultados de la medición de las variables. La información descriptiva se encuentra en la sección de anexos.

\section{Análisis estadístico}

Las diferencias entre las muestras (control y experimental), fueron establecidas a través del software de análisis estadístico SPSS versión 24. La prueba estadística Kolmogorov Smirnov fue empleado para determinar si la data tenía una distribución normal. Se realizaron otros análisis estadísticos para el estudio de la relación entre el efecto del baño químico y los días de almacenamiento, se utilizó la prueba de estadística inferencial Kruskal Wallis, con un nivel de significancia de 0,05. Para determinar la significancia entre las características sensoriales y la temperatura de almacenamiento se utilizó la prueba estadística de U de Mann Whitney, con un nivel de significancia de 0.05 .

Se utilizó estadística inferencial no paramétrica para probar cada una de las hipótesis.

Tabla No.2. Pruebas no paramétricas realizadas durante el estudio

\begin{tabular}{|c|c|c|c|}
\hline Hipótesis & $\begin{array}{c}\text { Estadístico de } \\
\text { prueba }\end{array}$ & $\begin{array}{c}\text { Significanci } \\
\mathbf{a}\end{array}$ & Resultado \\
\hline $\begin{array}{c}\text { HO }=\text { Tipo de } \\
\text { variable (control } \\
\text { y experimental). }\end{array}$ & $\begin{array}{l}\text { Kolmogorov- } \\
\text { Smirnov }\end{array}$ & $\mathrm{p}=0.000$ & $\begin{array}{c}\text { Existen diferencias } \\
\text { estadísticamente } \\
\text { significativas entre las } \\
\text { medidas del grupo control y } \\
\text { del grupo experimental. }\end{array}$ \\
\hline $\begin{array}{c}\mathrm{HO}_{2} \text { : Baño } \\
\text { químico (1-2-3). }\end{array}$ & $\begin{array}{l}\text { Kolmogorov- } \\
\text { Smirnov }\end{array}$ & $\mathrm{p}=0.000$ & $\begin{array}{l}\text { Existen diferencias } \\
\text { estadísticamente } \\
\text { significativas entre el baño } \\
\text { químico } 1,2 \text { y } 3 .\end{array}$ \\
\hline $\begin{array}{c}\mathrm{HO}_{3}: \text { Grados de } \\
\text { temperatura }\left(4^{\circ} y\right. \\
\left.8^{\circ}\right) .\end{array}$ & $\begin{array}{l}\text { Kolmogorov- } \\
\text { Smirnov }\end{array}$ & $\mathrm{p}=0.000$ & $\begin{array}{c}\text { Existen diferencias } \\
\text { estadísticamente } \\
\text { significativas entre los } \\
\text { grupos sometidos agrados de } \\
\text { temperatura } 4^{\circ} \text { y } 8^{\circ} \text {. }\end{array}$ \\
\hline $\begin{array}{l}\text { H05: Día }(4,8, \\
\text { 12). }\end{array}$ & $\begin{array}{l}\text { Kolmogorov- } \\
\text { Smirnov }\end{array}$ & $\mathrm{p}=0.000$ & $\begin{array}{l}\text { Existen diferencias } \\
\text { estadísticamente }\end{array}$ \\
\hline
\end{tabular}




\begin{tabular}{|c|c|c|c|}
\hline & & & $\begin{array}{l}\text { significativas por día }(4,8 \text {, } \\
12) \text {. }\end{array}$ \\
\hline HO6: Masa & $\begin{array}{l}\text { Kolmogorov- } \\
\text { Smirnov }\end{array}$ & $\mathrm{p}=0.000$ & $\begin{array}{c}\text { Existen diferencias } \\
\text { estadísticamente } \\
\text { significativas con respecto a } \\
\text { la masa }\end{array}$ \\
\hline $\begin{array}{l}\text { H07: Control } 1= \\
\text { Control } 2= \\
\text { Control } 3 \text { con } \\
\text { respecto a la } \\
\text { masa }\end{array}$ & Kruskal Wallis & $p=0.182$ & $\begin{array}{c}\text { No existen diferencias } \\
\text { estadísticamente } \\
\text { significativas, entre el grupo } \\
\text { control1, } 2 \text { y } 3 \text { respecto a la } \\
\text { masa }\end{array}$ \\
\hline $\begin{array}{l}\text { H0s: Muestra } A \\
=\text { Muestra } B= \\
\text { Muestra } C \text { con } \\
\text { respecto a la } \\
\text { masa }\end{array}$ & Kruskal Wallis & $\begin{array}{c}\text { valor } p= \\
0.969\end{array}$ & $\begin{array}{l}\text { No existen diferencias } \\
\text { estadísticamente } \\
\text { significativas, entre la } \\
\text { Muestra A, B y C con } \\
\text { respecto a la masa. } \\
\end{array}$ \\
\hline $\begin{array}{c}\text { H09: Baño } \\
\text { químico } 1 \text { = } \\
\text { Baño químico } 2 \\
=\text { Baño químico } \\
3 \text { con respecto a } \\
\text { la masa } \\
\end{array}$ & Kruskal Wallis & $p=0.466$ & $\begin{array}{l}\text { No existen diferencias } \\
\text { estadísticamente } \\
\text { significativas, entre el baño } \\
\text { químico } 1,2 \text { y } 3 \text { con } \\
\text { respecto a la masa. }\end{array}$ \\
\hline $\begin{array}{l}H_{10}: 4 \text { grados }= \\
8 \text { grados con } \\
\text { respecto a la } \\
\text { masa }\end{array}$ & U de Mann Whitney & $\mathrm{p}=0.105$ & $\begin{array}{l}\text { No existen diferencias } \\
\text { estadísticamente } \\
\text { significativas entre las } \\
\text { muestras expuestas a } 4 \text { y } 8 \\
\text { grados de temperatura con } \\
\text { respecto a la masa. }\end{array}$ \\
\hline $\begin{array}{c}\text { H011: Día } 1= \\
\text { Día } 4 \text { = Día } 8= \\
\text { Día } 12 \text { con } \\
\text { respecto a la } \\
\text { masa }\end{array}$ & Kruskal Wallis & $\mathrm{p}=0.000$ & $\begin{array}{c}\text { Existen diferencias } \\
\text { estadísticamente } \\
\text { significativas con respecto al } \\
\text { día de medición con } \\
\text { respecto a la masa. }\end{array}$ \\
\hline $\mathrm{H}_{13}: \mathrm{r}=0$ & Rho de Spearman & $\mathrm{p}=0.000$ & $\begin{array}{c}\text { Existe una relación positiva } \\
\text { y directa con un coeficiente } \\
\text { de correlación entre el grupo } \\
\text { control y el grupo } \\
\text { experimental de } 0.596\end{array}$ \\
\hline
\end{tabular}

Las pruebas estadísticas indican que sí hay diferencias significativas entre los baños químicos 1,2 y 3, siendo el más efectivo el baño químico 3, el cual tenía una concentración de $1.5 \mathrm{gL}-1$ de ácido cítrico y $1.5 \mathrm{gL}-1$ ácido ascórbico; se evidencia por baja presencia de pardeamiento enzimático en Persea americana. Con relación al tiempo de almacenamiento el análisis estadístico inferencial indica que si hay diferencias significativas por los días de almacenamiento, resultando que el mejor día fue el día 8. 

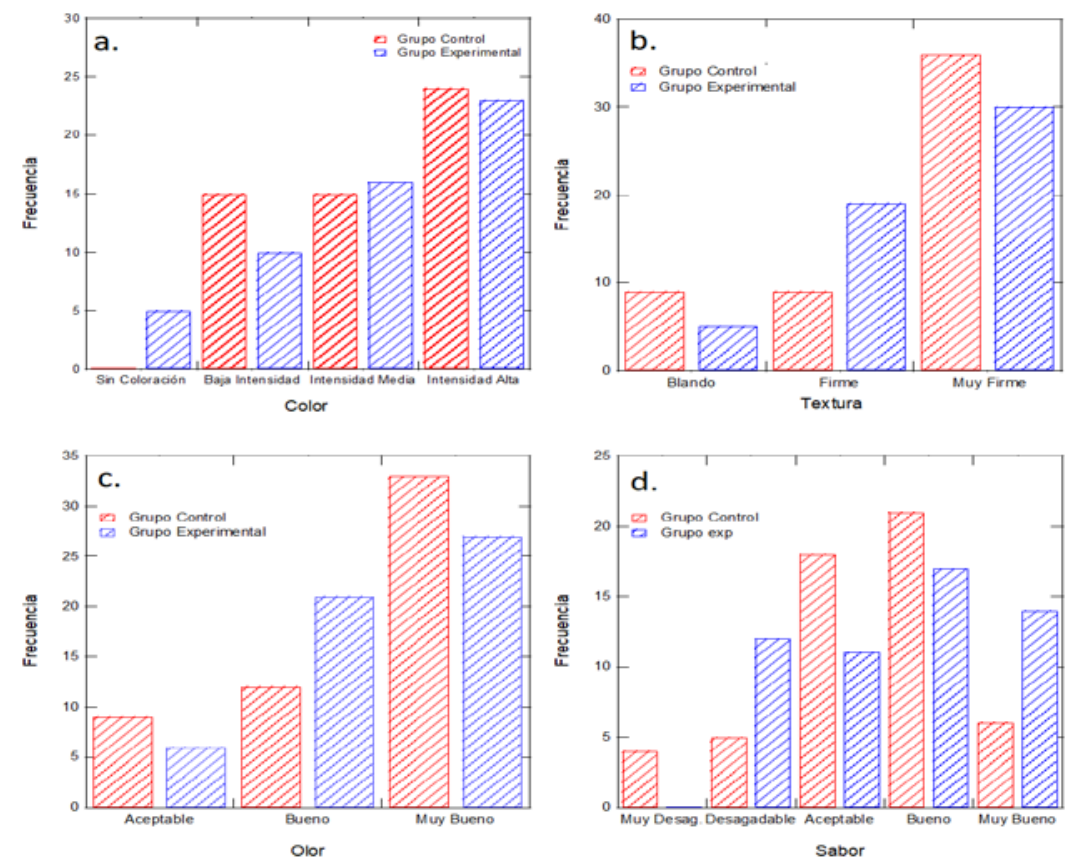

Figura No. 1. Resultados cualitativos para cuatro indicadores de calidad en el aguacate (color, textura, olor y sabor)

Estos resultados se obtuvieron a través de la evaluación que proporcionaron 9 participantes experimentales seleccionados con doble ciego. En el caso del color, los evaluadores proporcionaron mejores puntajes a las piezas en la intensidad media que pertenecen al grupo experimental (panel 1 de la Fig. 1). Los participantes consideraron que la magnitud de firmeza es mayor en el grupo experimental, aunque menor en la opción de respuesta "muy firme”. Se desconoce la rugosidad de la piel de los participantes y su capacidad def la medición al tacto. (panel B de la Figura 1). En el caso del olor, la evaluación con respecto al aroma tuvo niveles más elevados en el grupo control, (panel C de la figura 1). Finalmente se determinó que el sabor es muy bueno en el grupo experimental (panel D de la Figura 1).

El alcance de este estudio revela que, aunque existen instrumentos de laboratorio específicos y eficientes para medir el color, la textura, el olor y el sabor, para el equipo de investigación era interesante conocer la evaluación del consumidor final.

Ninguno de los participantes tuvo contacto o comunicación entre sí, antes y durante el experimento, de manera que sus respuestas estuvieran lo menos sesgadas posible. Se establecieron además los parámetros dictados en la Declaración Universal sobre Bioética y Derechos Humanos de la Organización de las Naciones Unidas. 


\section{Conclusión}

El aguacate ha sido objeto de numerosas investigaciones a lo largo de los años, sin embargo, es en la actualidad cuando se ha encontrado mayor interés científico no solamente debido a sus cualidades nutricionales y farmacéuticas. Se busca mejorar la calidad y aumentar la producción y consumo.

El objetivo en este trabajo estuvo encaminado a evaluar el efecto del baño químico con ácido cítrico y ácido ascórbico sobre el pardeamiento enzimático en Persea americana mínimamente procesada. El estudio se llevó a cabo con 108 porciones de aguacate, de los cuales el 50\% corresponde al grupo control y el resto al grupo experimental.

Las muestras son consistentes con relación a la masa, no hay evidencia estadística que la aplicación de los baños químicos y las temperaturas de almacenamiento influya en la pérdida de masa. En estudios similares realizados con fresas, los frutos recubiertos con los baños químicos perdieron menos peso (Gil-Giraldo et al., 2019), esto se puede explicar ya que en el estudio citado el baño químico contenía cloruro de calcio que tapona la entrada y salida de agua de los frutos. Con respecto a la relación de pérdida de masa y los días de almacenamiento se observa que a partir del día 4 hay evidencia que se inicia una pérdida de masa significativa.

El estudio demuestra que no existen diferencias significativas con valores $\mathrm{p}>0,05$ entre los distintos tipos de baños químicos con relación al olor, sabor, color, y textura; otros estudios sugieren que la actividad antioxidante de los ácidos orgánicos se manifiesta con concentraciones superiores al 25 por ciento masa/volumen (Baranco Contreras, 2003).

Los resultados establecen que existen diferencias significativas entre el olor, sabor y color y las temperaturas empleadas en el almacenamiento. El estudio demuestra que Persea americana sabe mejor a $8^{\circ} \mathrm{C}$.

El efecto del baño químico con ácido cítrico y ácido ascórbico sobre el pardeamiento enzimático no logró los resultados esperados con respecto a la textura y al olor, debido a que la evaluación estuvo a cargo del consumidor final, es decir de un panel de participantes seleccionados de manera probabilística aleatoria simple.

Estos alcances, aunque incipientes, son útiles porque motivan a continuar investigando el tema con la certeza de que se tomarán mejores decisiones metodológicas, además de contribuir con la seguridad alimentaria, en un futuro cercano, y al mismo tiempo determinar nuevas formas de preservar y comercializar este producto.

\section{Agradecimientos}

Gracias a todas las personas que han colaborado en este estudio; así como al equipo de investigación de la Universidad Especializada de las 
Américas. Se reconoce la orientación recibida para el diseño inicial de este manuscrito, a través del Seminario-Taller de Redacción de Artículos Científicos ofrecida por el Decanato de la Facultad de Biociencias y Salud Pública (junio de 2021).

\section{References:}

1. Arias, F., Montoya, C., \& Velásquez, O. (2018). Dinámica del Mercado mundial de aguacate. Revista Virtual Universidad Católica del Norte, (55), 22 -35.

2. Baranco Contreras, F. J (2003). Extracción y Evaluación de Compuestos con Capacidad Inhibitoria del Proceso de Obscurecimiento Enzimático de Aguacate Hass Persea americana variedad mill, obtenidos de la semilla del fruto. Tesis de ingeniero en ciencia y tecnología de alimentos. Universidad Autónoma Agraria “Antonio Narro” División de Ciencia Animal. Buenavista, Saltillo, Coahuila, México

3. Benen J. A, \& Visser J. (2003) Polygalacturonases. In: Whitaker J, Voragen A, Wong D (eds) Handbook of food enzymology. Marcel Gekker Inc, Nueva York, pp 857-866

4. Campos M., \& Tony B. (2014). Efecto del ácido cítrico en la inhibición del oscurecimiento enzimático de la yuca blanca (manihot esculenta crantz) mínimamente procesada, envasada y almacenada en refrigeración. Tesis de Ingenieria Agroindustrial. Facultad de Ingeniería y Ciencias Ambientales. Universidad Nacional Intercultural de la Amazonia. Departamento de Ucayali.

5. Cox K. A., McGhie, T. K., White, A. \& Woolf, A. B. (2004) Skin colour and pig-ment change during ripening of Hass avocado fruit. Posthar-vest Biology and Technology 31, 287-294.

6. Cortés-Herrera, C., Chacón, A., Artavia, G., Granados-Chinchilla, F., \& Pacifico, S. (2019). Simultaneous LC/MS Analysis of Carotenoids and Fat-Soluble Vitamins in Costa Rican Avocados (Persea americana Mill.). Molecules, 24(24), 4517. https://doi.org/10.3390/molecules24244517

7. Díaz, J., Ardila, C., \& Guerra, M. A. (2019). Case Study on the Eligibility of Colombian Hass Avocado in the US Market: Opportunities in East Asia. Online Journal Mundo Asia Pacífico, 8 (14), 5-27. DOI: 10.17230/map.v8.i14.01

8. FAO (2021). Organización de las Naciones Unidas para la alimentación y la agricultura. Aguacate. http://www.fao.org/faostat/es/\#search/aguacate

9. Gil-Giraldo, E. J., Duque-Cifuentes, A. L., \& Quintero-Castaño, V. D. (2019). Efecto del baño químico sobre la conservación de propiedades 
fisicoquímicas, microbiológicas y sensoriales de fresa. Biotecnología en el Sector Agropecuario y Agroindustrial, 17(2), 36-45. https://doi.org/10.18684/bsaa.v17n2.1251

10. Hernández, E. \& Briceño, L (2009). Evaluación del pardeamiento enzimático durante el almacenamiento en congelación del puré de Palta (Persea americana Mill) Var. Hass. Anales Científicos, UNALM, 70 (4), $1-12$

11. Herrera-González, J., Salazar-García, S., Martínez-Flores, H., \& RuizGarcía, J. (2017). Preliminary signs of physiological maturity and postharvest performance of Méndez avocado fruit. Revista Fitotecnia Mexicana, 40 (1), 55 - 63.

12. Mahendran T., Brennan J.G. \&Hariharan G. Aroma volatiles components of "Fuerte" Avocado (Persea americana Mill.) stored under different modified atmospheric conditions. Journal of Essential Oil Research. 2019;31(1):34-42. doi:10.1080/10412905.2018.1495108

13. Minh, N. P. (2020). Evaluation of physicochemical and antioxidant characteristics of spray dried avocado (Persea americana) powder under storage. Research on Crops, 21(4), 687-692. https://doi.org/10.31830/2348-7542.2020.107

14. Papagianni, M., Nokes, S., \& nFiler, K. (2001). Submerged and solidstate phytase fermentation by Aspergillus niger: Effects of agitation and medium viscosity on phytase production, fungal morphology and inoculum performance. Food Tech. Biotechn. 39 (4), 319-326.

15. Restrepo Osorio, C., Gómez Velásquez, F. A., Correal, A. G., Torres Bonilla, J. M., \& Urrea Trujillo, A. I. (2018). In vitro propagation of avocado (Persea americana Mill.) cv. Hass through morphogenesis. Acta Agronómica, 67(1), 160-167. https://doi.org/10.15446/acag.v67n1.61474

16. Rosero, J. C., Cruz, S., Osorio, C., Hurtado, N., \& Moreno, D. A. (2019). Analysis of Phenolic Composition of Byproducts (Seeds and Peels) of Avocado (Persea americana Mill.) Cultivated in Colombia. Molecules, 24(17), 3209. https://doi.org/10.3390/molecules24173209

17. Salgado-Cervantes, M., Servent, A., Maraval, I., Vargas-Ortiz, M., \& Pallet, D. (2019). Flash Vacuum-Expansion Process: Effect on the Sensory, Color and Texture Attributes of Avocado (Persea americana) Puree. Plant Foods for Human Nutrition (Dordrecht, Netherlands), 74(3), 370-375. https://doi.org/10.1007/s11130-019-00749-3

18. Talavera, A., Soorni, A., Bombarely, A., Matas, A. J., \& Hormaza, J. I. (2019). Genome-Wide SNP discovery and genomic characterization in avocado (Persea americana Mill.). Scientific Reports, 9(1), 1-13. https://doi.org/10.1038/s41598-019-56526-4 
19. Vergara-Pulgar, C., Rothkegel, K., González-Agüero, M., Pedreschi, R., Campos-Vargas, R., Defilippi, B. G., \& Meneses, C. (2019). De novo assembly of Persea americana cv. "Hass" transcriptome during fruit development. BMC Genomics, 20(1), 1-14. https://doi.org/10.1186/s12864-019-5486-7

20. Xiao, L. A. \& Kiyota, M. (2000). Design of modified atmosphere package for retaining freshness of avocado (Persea americana) fruits. Environ. Control in Biol. 38: 157-164.

21. Zhi Hung , L. \& Yau Yan, L. (2018). Drying effects on antioxidant activity, enzyme activity, and phytochemicals of avocado (Persea americana) leaves. Journal of Food Processing and Preservation, 42(10), e13667. https://doi.org/10.1111/jfpp.13667 